FOLIA

Amazónica

Revista del Instituto de Investigaciones

de la Amazonía Peruana

\title{
CARACTERIZAÇÃO QUÍMICA E ATIVIDADE ANTIFÚNGICA DOS ÓLEOS ESSENCIAIS DE LARANJA-KINKAN (Fortunella margarita (LOUR.) SWINGLE)
}

\author{
Antonio Carlos PEREIRA DE MENEZES FILHO ${ }^{1}$, \\ Carlos Frederico DE SOUZA CASTRO ${ }^{1}$ \\ 1 Instituto Federal de Educação, Ciência e Tecnologia Goiano, Campus Rio Verde, Rio Verde, Goiás, \\ Brasil. Correio electrónico: astronomoamadorgoias@gmail.com
}

\section{RESUMO}

A laranja-kinkan (Fortunella margarita) é bem conhecida pelo fruto comestível e rico em antioxidantes, mas muitos outros usos benéficos ainda precisam ser investigados. Neste estudo, empregamos cromatografia gasosa com espectrometria de massas (CG-MS), para avaliar o potencial antifúngico dos óleos essenciais dos frutos verde e maduro para inibir efetivamente os patógenos vegetais: Colletotrichum acutatum, C. gloeosporioides e Sclerotinia sclerotiorum. Frutos verdes e maduros de F. margarita foram coletados em uma unidade rural no município de Rio Verde, Goiás, Brasil. Adicionalmente, o rendimento de óleo essencial foi determinado por hidrodestilação em sistema tipo Clevenger. Foram obtidos rendimentos de óleos essenciais de 3,57 e 3,64\% para os frutos verde e maduro. No perfil químico foram observados seis compostos majoritários $\beta$-pineno, limoneno, $\beta$-felandreno, $\alpha$-guaieno, germacreno D e biciclosesquifelandreno para ambas as amostras. Os óleos essenciais apresentaram alta eficiência no percentual de inibição contra os três patógenos fúngicos. Portanto, os óleos essenciais dos frutos verde e maduro da laranja-Kinkan podem ter aplicações antifúngicas.

PALAVRAS-CHAVE: Colletotrichum gloeosporioides, C. acutatum, Sclerotinia sclerotiorum, atividade fungistática. 


\title{
CHEMICAL CHARACTERIZATION AND ANTIFUNGAL ACTIVITY OF ESSENTIAL OIL OF ORANGE KINKAN (Fortunella margarita (LOUR.) SWINGLE)
}

\begin{abstract}
Orange Kinkan (Fortunella margarita) is well known for its edible and antioxidant rich fruits. In this study, we employed Gas chromatography-mass spectrometry (GC-MS) to evaluate the antifungal potential of essential oils within both green and ripe fruits to effectively inhibit the following common plant pathogens: Sclerotinia sclerotiorum, Colletotrichum gloeosporioides, and Colletotrichum acutatum. Green and ripe fruits of cultivated Orange Kinkan were collected from a rural unit in Rio Verde, Goiás, Brazil. Additionally, the essential oil yield was determined by hydrodistillation in Clevenger-type apparatus. We observed essential oil yields of 3.57 and $3.64 \%$ to green and ripe fruits. Within the essential oils, we detected six major compounds $\beta$-pinene, limonene, $\beta$-felandrene, $\alpha$-guaiene, germacrene $D$, and bicyclosesquifellandrene. Essential oils showed high inhibition percentage efficiency against the three fungal pathogens. Therefore, the essential oils of the green and ripe fruits of orange Kinkan may have antifungal applications.
\end{abstract}

KEYWORDS: Colletotrichum gloeosporioides, C. acutatum, Sclerotinia sclerotiorum, anti-fungal. 


\section{INTRODUÇÃO}

Os citros são costumeiramente utilizados para produção de sucos e doces, bem como para uso ornamental. 0 uso remota por volta de 1000 a.C. no continente Asiático nas regiões chinesas (Mazzini \& Pio, 2010). Nas Américas os citros foram introduzidos durante a colonização portuguesa e espanhola a partir de 1530, e o início para produção agrícola em larga escala deu-se a partir de 1600 (Donadio et al., 2005).

O Brasil ocupa lugar de destaque sendo o primeiro maior produtor de cítricos do mundo, onde sua maior parcela de produção é direcionada para plantas industriais de sucos tipo exportação. O estado de São Paulo - Brasil, ocupa a primeira posição de maior produtor de citros com cerca de $85 \%$ da parcela de produção brasileira anual (Lopes et al., 2011).

As espécies cítricas do gênero Citrus pertencem à família Rutaceae, sendo nativa do sudoeste asiático, com dispersão filogenética desde a China ao Japão, Índia a Nova Guiné e da Austrália a África tropical (Diniz \& De Oliveira, 2015; Sicari \& Poiana, 2017). A espécie Fortunella margarita apresenta porte arbóreo pequeno, florescendo na primavera e verão apresentando flores de cor branca com leve aroma adocicado, e frutos no outono. Os frutos inicialmente apresentam coloração verde e quando maduros tonalidade fortemente alaranjada (Donadio et al., 2005). Os frutos de F. margarita (Lour.) Swingle apresentam características comuns das frutas cítricas, como aroma e sabor ácido, entretanto, contém menor quantidade de gomos, bem como, casca mais fácil de digerir, podendo ser empregada ainda na forma in natura ou na produção de doces em caldas e cristalizados (Rocha \& Soares Filho, 2009; Diniz \& De Oliveira, 2015). A casca apresenta compostos aromáticos pertencentes à classe dos óleos essenciais (OEs).
Os OEs são constituídos basicamente por compostos voláteis por monoterpenos, diterpenos, sesquiterpenos e fenilpropanóides produzidos naturalmente a partir do metabolismo secundário dos vegetais. Esses compostos apresentam aromas que muitas das vezes são produzidos com intuito de polinização, contra animais herbívoros e ataques de insetos. Os compostos de OEs podem ser produzidos por diversos órgãos vegetais como flores, folhas, frutos, talos, galhos, raízes, cascas e sementes, sendo comercializados para produção de perfumes, fragrâncias, medicamentos e agentes agrícolas (De Morais et al., 2006; SolórzanoSantos \& Miranda-Novales, 2012; Miranda et al., 2016).

Os compostos voláteis apresentam importantes ações farmacológicas, agrícolas, biológicas e alimentícias. Vários OEs apresentam importante atividade antioxidante, antitumoral, leishmanicida, moluscicida, inseticida, acaricida, antibacteriano, antiviral e principalmente ação fungicida (Chagas et al., 2002; Nascimento et al., 2007; Pombo et al., 2018).

0 uso de agentes antifúngicos aplicados na agricultura provém de formulados químicos sintéticos, que muitas das vezes são utilizados de forma incorreta, e durante ao longo do ano em diferentes fases do desenvolvimento do vegetal. Com isso, vem provocando o aparecimento de espécies fúngicas resistentes a estes antifúngicos comerciais. Além disso, compostos sintéticos apresentam alta toxicidade para o ambiente e o ser humano, visto que, há a contaminação dos solos, da água e das formas de vida, principalmente dos insetos polinizadores (Dann et al., 2002; Cruz et al., 2010; Da Silva et al., 2018).

Atualmente vem-se pesquisando o uso de formas alternativas aos produtos sintéticos, a base de extratos e OEs que apresentem ação fungicida equiparável aos produtos sintéticos no mercado. Os produtos naturais não apresentam toxicidade 
para as formas de vida e não há contaminação do solo e da água quando aplicados, assim não há danos ao meio biótico (De Souza et al., 2019).

o bioma Amazônico é considerado o berço de inúmeras espécies fúngicas para a região, devido aos excelentes fatores bióticos (Araújo \& Sousa, 1998). Fungos fitopatológicos apresentam o maior índice em percentagem de infecção em espécies frutíferas nas principais famílias Annonaceae, Araceae, Cannaceae, Fabaceae, Heliconiaceae, Malphiguiaceae, Moraceae, Myrtaceae, Passifloraceae, Poaceae, Rubiaceae e Sapotaceae. Os países além do Brasil, como Peru, Bolívia, Ecuador, Colômbia, Guiana, Guiana Francesa, Suriname e Venezuela, fazem parte da Amazônia internacional, onde a produção de frutos para importação e exportação apresenta porcentagem importante do PIB do país, onde são afetados constantemente por fitopatologias que causam perda considerável de produção gerando grande déficit agrícola todos os anos (Do Carmo et al., 2016). Alguns exemplos de infecção fúngica foram observados em 1982 e 1987 com os primeiros casos registrados de Sigatoka negra (Mycosphaerella fijiensis) em bananais da Colômbia, Ecuador e Venezuela (Mendes et al., 2007). No açaizeiro pela antracnose cauda pelo Colletotrichum gloeosporioides, principalmente no estado do Acre, Brasil (Monteiro et al., 2017). No cacau e cupuaçu por Moniliophthora roreri causador da monilíase sendo identificada em plantações no Perú e Bolívia (Siviero et al., 2019).

Em uma visão macroscópica de produção, os fungos fitopatológicos causam expressivas perdas também nas áreas agricultáveis de soja, milho, sorgo, algodão, grão-de-bico, feijão e amendoim (Ferreira \& Warren, 1982; Boland \& Hall, 1994; Amselem et al., 2011; Bragança et al., 2019; Damm et al., 2019).

A fauna micológica como agentes fitopatogênicos é rica em espécies agrícolas, sendo o mofo-branco (Sclerotinia sclerotiorum), e as diversas formas de antracnose principalmente do Colletotrichum acutatum e C. gloeosporioides que a cada ano geram consideráveis perdas agrícolas na produção de grãos e de frutas, gerando na cadeia produtiva, grandes perdas econômicas aos pequenos, médios e grandes produtores rurais, bem como ao consumidor brasileiro.

Este estudo teve por objetivo, avaliar o perfil químico do óleo essencial dos frutos verde e maduro de Fortunella margarita, bem como a atividade antifúngica frente à Colletotrichum acutatum e C. gloeosporioides e Sclerotinia sclerotiorum.

\section{MATERIAL E MÉTODOS}

Os frutos verde e maduro de laranja-kinkan foram coletados em uma propriedade rural no município de Rio Verde, Goiás, Brasil, com as seguintes coordenadas geográficas: $17^{\circ} 43^{\prime} 09.4^{\prime \prime} \mathrm{S}$ $50^{\circ} 53^{\prime} 06.5^{\prime \prime} \mathrm{W}$. No laboratório, os frutos foram descascados com ajuda de uma faca inox. 0 material foi pesado e processado em alíquotas de 100 g de casca in natura. A solução obtida foi separadamente transferida para um sistema tipo Clevenger. 0 sistema ficou em refluxo por $3 \mathrm{~h}$. Em seguida, o hidrolato foi coletado e transferido para um funil de separação, onde foi lavado três vezes com $30 \mathrm{~mL}$ de diclorometano (P.A - ACS). Logo após, as frações foram coletadas e secas com sulfato de sódio anidro (P.A - ACS). O filtrado foi coletado e transferido para béqueres envoltos com papel alumínio. A solução foi mantida em local seco e ao abrigo da luz até completa evaporação do solvente. Em seguida, os OEs foram pesados e o rendimento determinado em porcentagem. 0 rendimento foi calculado conforme equação, Rendimento $(\%)=$ (massa de óleo essencial) massa de material vegetal)*100.

O perfil químico dos OEs de Fortunella margarita, foi determinado por cromatografia 
gasosa com espectrometria de massas (CG-EM) (Shimadzu GC Mod. QP 5000), coluna capilar de sílica fundida (Optima ${ }^{\circledR}, 5-0,25 \mu \mathrm{m}(30 \mathrm{~m} \mathrm{x}$ 0,25 mm)), com espectrômetro de massas por ionização em impacto eletrônico (IE) (70 e.V). A identificação dos compostos químicos do óleo essencial foi baseada através do Índice de Kovats, utilizando uma série de $n$-alcanos (C-1 a C-40). A comparação do tempo de retenção foi realizada utilizando a espectroteca Nist 11 e literatura Adams (2007).

0 teste antifúngico foi realizado utilizando isolados de Colletotrichum acutatum e $C$. gloeosporioides e Sclerotinia sclerotiorum, mantidos em meio BDA. As cepas foram doadas pelos laboratórios de Química de Produtos Naturais e Química Tecnológica do Instituto Federal Goiano, campus Rio Verde. A atividade antifúngica dos OEs sobre o crescimento micelial de S. sclerotiorum, C. gloeosporioides e C. acutatum, foi avaliado através de diferentes concentrações, partindo de 100 (óleo puro); 50; 25; 12,5; 6,25; 3,13 e 1,56 $\mu \mathrm{L} \mathrm{mL}^{-1}$ diluídas em dimetilsulfóxido (DMSO). Como controle negativo, utilizou-se a testemunha (ausência de OE), fungicida comercial Frowncide $^{\circledR}$, na concentração de $10 \mu \mathrm{L} \mathrm{mL}^{-1}$ e DMSO como controle positivo.

As concentrações dos OEs foram adicionadas ao meio de cultura BDA após esterilização e resfriamento, bem como para os tratamentos com fungicida comercial e DMSO. Após solidificação do meio, em câmara de fluxo laminar, 1 disco de micélio para cada cepa de $C$. acutatum, $C$. gloeosporioides e $S$. sclerotiorum, com $9 \mathrm{~mm}$ de diâmetro, foi depositado no centro da placa de Petri de $9 \mathrm{~cm}$ de diâmetro. Em seguida foram incubadas à temperatura entre 20,23 e $25{ }^{\circ} \mathrm{C}$ respectivamente, conforme descrito por Cunico et al. (2002), Garcia et al. (2012) e Santos et al. (2012), com adaptações.

A avaliação consistiu em medições diárias do diâmetro das colônias, por meio de um paquímetro manual, iniciadas após 24 horas do início da incubação e encerradas, quando as colônias fúngicas, do tratamento testemunha, atingiram completamente a área interna da placa. A determinação do percentual de inibição de crescimento (PIC\%) foi realizada conforme proposto por Garcia et al. (2012). A equação utilizada foi PIC\% = (DTT - DTQ $) / D T T \times 100$. Onde: $\mathrm{PIC}=$ percentual de inibição de crescimento, DTT = diâmetro no tratamento testemunha, DTQ = diâmetro no tratamento químico.

A análise consistiu em uma avaliação em triplicata seguida de ( \pm ) desvio padrão. Para determinação da diferença estatística, foi adotado o teste de Tukey $(\mathrm{p} \leq 0,05)$. 0 software de estatística utilizado foi o PAST 3 (versão livre).

\section{RESULTADOS}

Os rendimentos de OE foram de 3,57 e 3,64\% para os frutos verde e maduro de Fortunella margarita, respectivamente. Na Tabela 1 estão descritos os perfis químicos dos OEs dos frutos verde e maduro de Fortunella margarita por CG-EM.

Na Figura 1 estão apresentados os resultados de porcentagem de inibição de crescimento pelo $\mathrm{OE}$ do fruto verde de $F$. margarita frente à $S$. sclerotiorum, C. gloeosporioides e C. acutatum.

Observa-se alta sensibilidade das cepas frente às concentrações de óleo essencial dos frutos verdes. Para S. sclerotiorum observa-se a formação de quatro grupos estatisticamente diferentes avaliados pelo teste de Tukey. Em todas as concentrações foi possível observar atividade de inibição micelial onde nas maiores concentrações foram observados os melhores resultados, para 25,50 e $100 \mu \mathrm{L}^{-1}$ com inibições entre 73,55 a $89,14 \%$.

Para Colletotrichum gloeosporioides houve a formação de dois grupos diferentes avaliados pelo teste de Tukey. A atividade de inibição foi 
Tabela 1. Perfis químicos por cromatografia gasosa com espectrometria de massas (CG-EM) dos óleos essenciais dos frutos verde e maduro de Fortunella margarita (laranja-Kinkan).

\begin{tabular}{|c|c|c|c|}
\hline Composto & $I R^{*}$ & $\mathrm{FV}^{* *}$ & $\mathrm{FM}^{* *}$ \\
\hline$\alpha$-pineno & 937 & 0,35 & 0,30 \\
\hline$\beta$-pineno & 980 & 20,19 & 11,42 \\
\hline$\beta$-mirceno & 985 & - & 1,17 \\
\hline Limoneno & 1032 & 15,12 & 20,76 \\
\hline$\gamma$-terpineno & 1042 & 2,76 & 2,01 \\
\hline Trans-arbusculone & 1072 & 4,12 & 4,00 \\
\hline Humulano-1,6-dien-3-ol & 1116 & 0,96 & 0,54 \\
\hline Cis-ocimenol & 1131 & 0,23 & 0,21 \\
\hline$\delta$-3-careno & 1150 & 0,19 & 0,60 \\
\hline Trans- $\beta$-ocimeno & 1153 & 0,27 & - \\
\hline$\alpha$-terpineol & 1183 & 0,66 & 0,98 \\
\hline$\beta$-felandreno & 1217 & 11,81 & 12,30 \\
\hline E- $\beta$-ocimeno & 1250 & 0,30 & 0,25 \\
\hline$\alpha$-cubebeno & 1346 & 0,27 & 0,28 \\
\hline Isoledeno & 1365 & - & 2,35 \\
\hline$\alpha$-copaeno & 1370 & 0,99 & - \\
\hline Oleato de geranil & 1377 & 0,37 & 0,34 \\
\hline Longifoleno & 1408 & 0,56 & 0,79 \\
\hline$\beta$-panasinseno & 1411 & 0,20 & - \\
\hline$\gamma$-elemeno & 1417 & - & 1,74 \\
\hline$\beta$-copaeno & 1423 & 3,55 & 0,42 \\
\hline$\alpha$-guaieno & 1430 & 6,14 & 5,88 \\
\hline Aromadendreno & 1439 & 4,16 & 1,68 \\
\hline Allo-amadendreno & 1453 & 0,28 & 0,23 \\
\hline Cis-muurola-4(14),5-dieno & 1473 & - & 0,85 \\
\hline Germacreno D & 1476 & 4,31 & 9,00 \\
\hline$\delta$-selineno & 1490 & 3,63 & 5,21 \\
\hline Epizonareno & 1508 & 1,09 & 0,38 \\
\hline Globulol & 1581 & 1,77 & 1,00 \\
\hline Terpinen-4-ol & 1597 & 0,29 & 0,36 \\
\hline Angelato de mirtenil & 1608 & 0,68 & 0,23 \\
\hline 10-Epi- $\gamma$-eudesmol & 1615 & 2,35 & 2,28 \\
\hline Epi-1-cubenol & 1619 & 0,12 & 0,33 \\
\hline Junenol & 1622 & 0,62 & 0,80 \\
\hline$\gamma$-muuroleno & 1681 & 3,62 & 2,11 \\
\hline Trans-ocimenol & 1685 & - & 0,19 \\
\hline Biciclosesquifelandreno & 1726 & 6,25 & 8,07 \\
\hline Selina-3,7(11)-dieno & 1791 & 0,44 & 0,89 \\
\hline Total identificado (\%) & & 98,65 & 99,95 \\
\hline
\end{tabular}

$\mathrm{IR}^{*}=$ Índice de Retenção literário (Adams, 2007). FV** = Fruto Verde e FM** = Fruto Maduro, em porcentagem de retenção do pico. 
Figura 1: Porcentagem de inibição de crescimento (PIC\%) do óleo essencial do fruto verde de Fortunella margarita frente à Colletotrichum acutatum, C. gloeosporioides e Sclerotinia sclerotiorum. Letras iguais não diferem estatisticamente entre si pelo teste de Tukey $(p \leq 0,05)$.

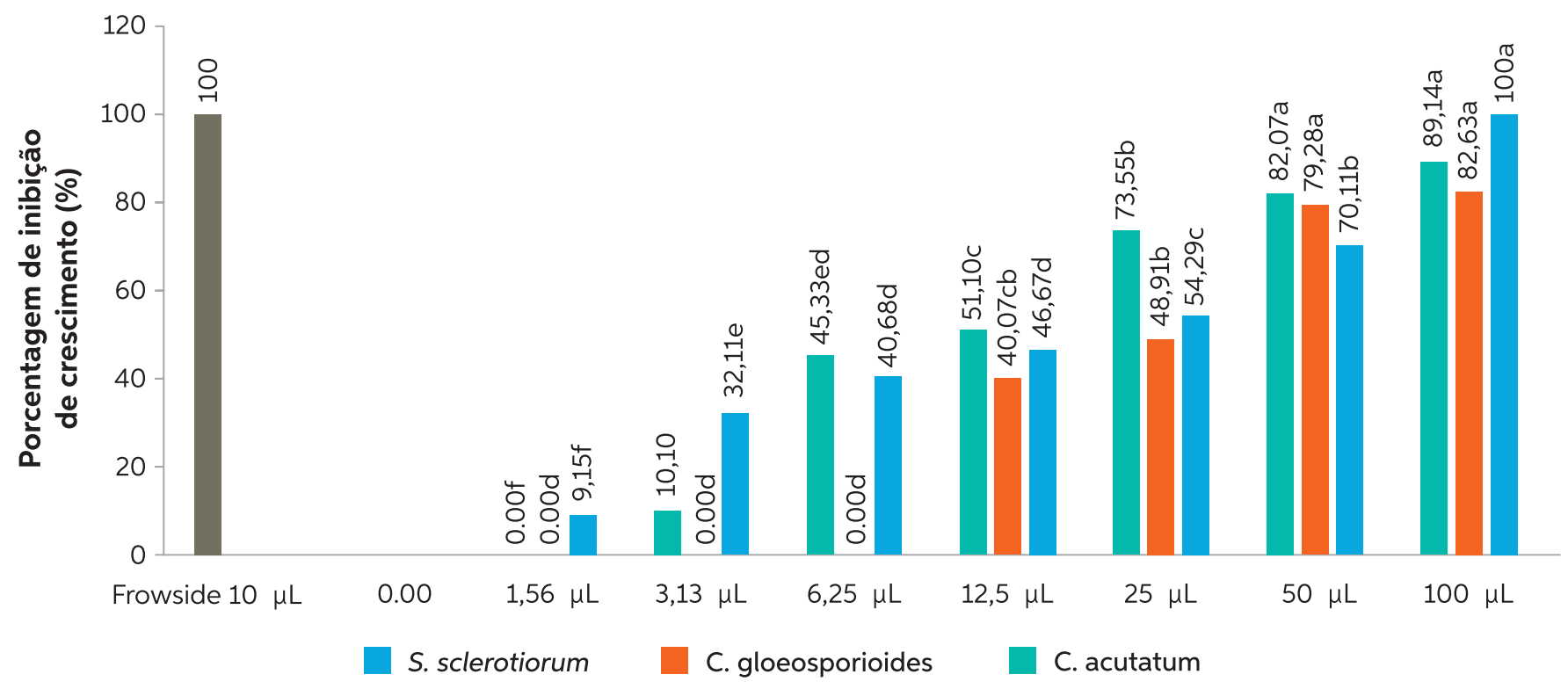

Figura 2: Porcentagem de inibição de crescimento (PIC\%) do óleo essencial do fruto maduro de Fortunella margarita frente à Colletotrichum acutatum, C. gloeosporioides e Sclerotinia sclerotiorum. Letras iguais não diferem estatisticamente entre si pelo teste de Tukey $(p<0,05)$.

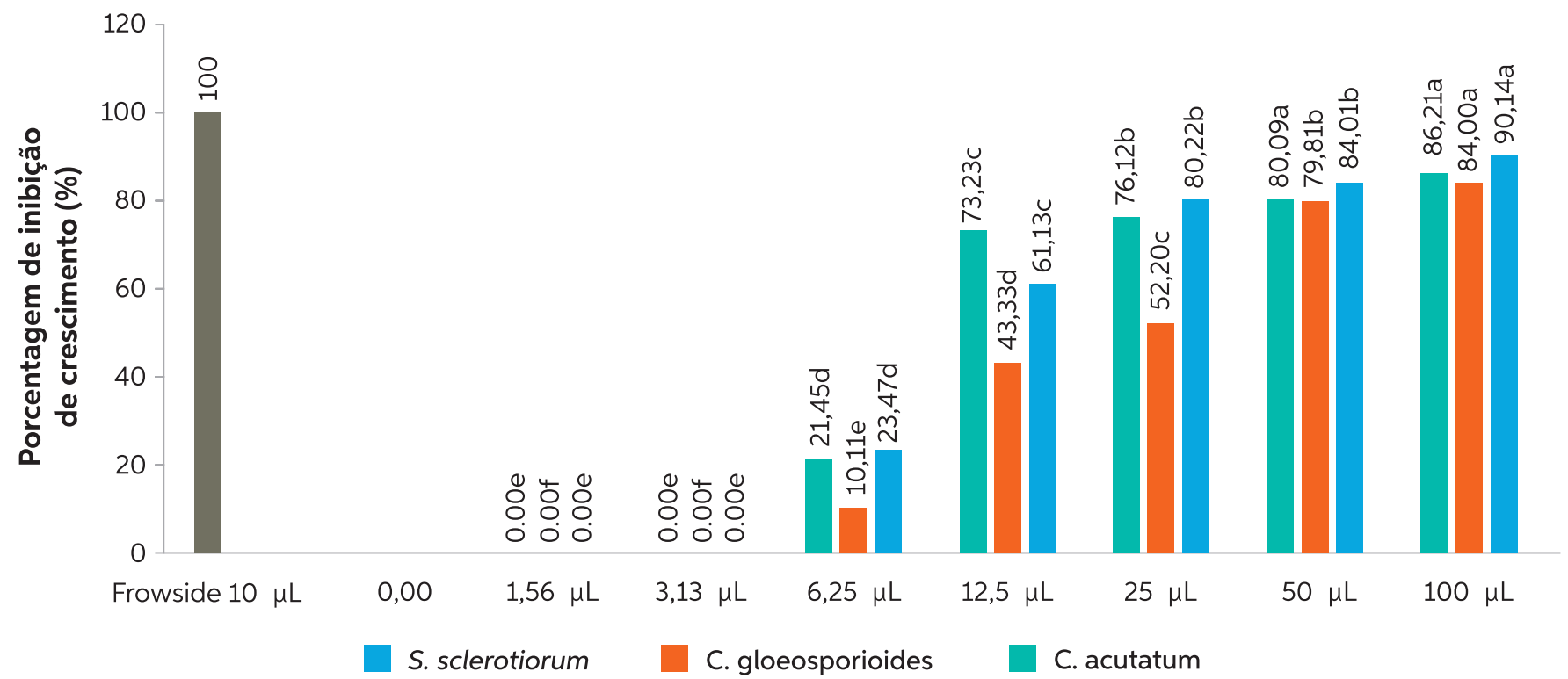


observada apenas nas maiores concentrações de 25,50 e $100 \mu \mathrm{L}^{-1}$ com resultados de 48,91; 79,28 e $82,63 \%$, respectivamente. Nas concentrações de 1,56; 3,13 e 6,25 $\mu \mathrm{L}^{-1}$ não houve inibição de crescimento para C. gloeosporioides (Figura 1). Já para C. acutatum foram observados a formação de seis grupos estatisticamente diferentes. Novamente as melhores taxas de inibição foram observadas nas maiores concentrações entre 25; 50 e $100 \mu \mathrm{L}^{-1}$ com taxa de inibição entre 54,29; 70,11 e 100\%. Para C. acutatum foram observados em todas as concentrações atividade de inibição de crescimento.

Na Figura 2 estão apresentados os resultados de atividade de inibição do crescimento micelial pelo $\mathrm{OE}$ do fruto maduro de F. margarita frente à C. acutatum, C. gloeosporioides e $S$. sclerotiorum.

\section{DISCUSSÃO}

Os OEs para os frutos verde e maduro de $F$. margarita apresentaram bom rendimento de extração igual a 3,57 e 3,64\%, respectivamente. Ambos OEs apresentaram coloração incolor e como característica sensorial, aroma típico do fruto. A coloração incolor e aroma foram também observados por Sicari \& Poiana (2017) avaliando os frutos de F. margarita. No estudo de Sicari \& Poiana (2017), os pesquisadores obtiveram rendimento de $\mathrm{OE}$ para a casca do fruto in natura e seca igual a 3,16 e 4,8\% respectivamente.

0 mesmo é observado para diferentes tipos de Citrus apresentados por Millezi et al. (2014) onde encontraram rendimento de 1,85\% para cascas do fruto de Citrus limonia. Lopes et al. (2013) obtiveram rendimentos entre 9,9 a $10,76 \%$ de OE das cascas de Citrus limettioides em diferentes localidades no estado de Goiás, Brasil. Botelho et al. (2009) obtiveram para cascas dos frutos de Citrus aurantium rendimento de OE de 0,37\%.
Foram observados seis compostos majoritários nos OEs dos frutos verde e maduro (Tabela 1). Sendo os compostos com maiores teores observados para $\beta$-pineno 20,19 e 11,42\%, limoneno 15,12 e 20,76\%, $\beta$-felandreno 11,81 e $12,30 \%$, $\alpha$-guaieno 6,14 e $5,88 \%$, germacreno D 4,31 e 9,00\% e biciclosesquifelandreno 6,25 e 8,07\%, respectivamente para frutos verde e maduro. 0 composto majoritário limoneno foi superior nos estudos propostos por Sicari \& Poiana (2017) com teor médio de 96,44\% extraído em quatro processos de extração, para Fitsiou et al. (2016) de 93,78\%, e Sutour et al. (2016) de 93,1\%. Estas médias superiores para limoneno nos frutos de F. margarita são observadas em outros estudos propostos por Koyasako \& Bernhard (1983), Quijano \& Pino (2009), Wang et al. (2012).

De acordo com Martins et al. (2006), vários fatores podem estar envolvidos quanto ao teor de um composto em OE, como local de coleta, fertilidade do solo, sazonalidade, idade do vegetal, radiação solar, pluviosidade, ataque de insetos, herbívoros, origem geográfica e genética.

Em outros estudos, a variação dos teores dos compostos majoritários para OEs do gênero Citrus que apresentam também variação em sua composição, como observados por Millezi et al. (2014) onde encontraram para C. limonia, o composto majoritário limoneno com teor de $33,67 \%$ e para $p$-cimeno com $14,16 \%$. De acordo com os pesquisadores citados anteriormente e por Ladaniya (2008), os vegetais do gênero Citrus podem apresentar teores de limoneno de até 80\%. Lopes et al. (2013) encontraram 18 compostos para o óleo essencial de $C$. limettioides, sendo o limoneno o composto majoritário com médias entre 70,60 a 75,18\%, o composto linalol apresentou médias entre 8,80 a 11,23\%. Já no estudo proposto por Botelho et al. (2009) os pesquisadores encontraram para o $\mathrm{OE}$ da laranja 
lima (Citrus aurantium) cerca de 20 compostos, sendo o limoneno o composto majoritário com $76,2 \%$. Os compostos com intensidade média foram mirceno com 5,52\% e linalol com 3,93\%.

Na Figura 1 observa-se alta sensibilidade das cepas sobre às concentrações de $\mathrm{OE}$ do fruto verde. Para S. sclerotiorum observa-se a formação de quatro grupos estatisticamente diferentes, avaliados pelo teste de Tukey. Em todas as concentrações foi possível observar atividade de inibição micelial de crescimento, onde nas maiores concentrações $\left(25,50\right.$ e $\left.100 \mu \mathrm{L}^{-1}\right)$ foram obtidos os melhores resultados, com inibições entre 73,55 a 89,14\%.

Para Colletotrichum gloeosporioides houve a formação de dois grupos que apresentaram diferença estatística pelo teste de Tukey. A atividade de inibição foi observada apenas nas maiores concentrações de 25, 50 e 100 $\mu \mathrm{L}^{-1}$ com resultados médios de 48,91; 79,28 e $82,63 \%$, respectivamente. Nas concentrações de 1,56; 3,13 e 6,25 $\mu \mathrm{L}^{-1}$ não houve inibição de crescimento para C. gloeosporioides (Figura 1). Já para $C$. acutatum foram observados seis grupos estatisticamente diferentes. Novamente as melhores taxas de inibição foram observadas nas maiores concentrações entre 25; 50 e $100 \mu \mathrm{L}^{-1}$ com inibição entre 54,29; 70,11 e 100\%. Para $C$. acutatum todas as concentrações de OE do fruto verde apresentaram atividade de inibição de crescimento (Figura 1).

Para a atividade antifúngica do $\mathrm{OE}$ fruto maduro é possível verificar também efetiva atividade de inibição de crescimento micelial para S. sclerotiorum, C. gloeosporioides e C. acutatum. Entretanto, quando comparados aos resultados de atividade micelial do $\mathrm{OE}$ fruto verde, os resultados foram superiores como observados para $S$. sclerotiorum e C. gloeosporioides, e inferiores para $C$. acutatum (Figura 2). Para S. sclerotiorum observou-se a formação de quatro grupos com diferença estatística pelo teste de Tukey. Para $C$. gloeosporioides houve a formação de cinco grupos, e para $C$. acutatum quatro grupos estatisticamente diferentes conforme avaliado pelo teste de Tukey.

Novamente observam-se as melhores atividades de inibição para as maiores concentrações de 12,5; 25; 50 e $100 \mu \mathrm{L}^{-1}$. Para $S$. sclerotiorum foram observados taxas de inibição entre 21,45 a 86,21\%; para C. gloeosporioides entre 10,11 a $84,00 \%$ e para $C$. acutatum de 23,47 a 90,14\% (Figura 2). Em comparação aos resultados obtidos em ambos os OEs e respectivas concentrações de inibição, foram comparadas entre si com o fungicida comercial Frowncide ${ }^{\circledR}$ na concentração de $10 \mu \mathrm{L}^{-1}$ onde apresentou inibição de $100 \%$ para as três cepas fúngicas avaliadas.

Os OEs dos frutos verde e maduro de $F$. margarita apresentaram alta eficiência no controle e inibição do crescimento micelial para as três cepas fúngicas avaliadas neste estudo. Como não foram avaliados os compostos majoritários individualmente, podemos predizer que todos os compostos trabalham em sinergismo promovendo a ação fungistática, o mesmo é discutido por Xavier et al. (2016).

Resultados promissores também foram observados por Silva et al. (2018), onde avaliaram o OE das folhas de Psidium guajava em dois períodos de coleta, com eficiência de inibição micelial frente a $S$. sclerotiorum, nas maiores concentrações de 300 e $200 \mu \mathrm{L}^{-1}$. Os pesquisadores encontraram porcentagens de inibição entre 94,9 a 90,0\% e na menor concentração de $100 \mu \mathrm{L}^{-1}$ com 80 a 77,5\%. No estudo de Xavier et al. (2016), os pesquisadores obtiveram porcentagem de inibição micelial em $S$. sclerotiorum entre 87,63 a $28,27 \%$ para as concentrações entre 300 a $50 \mu \mathrm{L}^{-1}$ com OE das folhas de Cardiopetalum calophyllum. Dias-Arieira et al. (2010), avaliaram a atividade antifúngica dos OEs de nim (Azadirachta indica) e de eucalipto (Eucalyptus citriodora) onde obtiveram inibição entre 74,4 a $84,4 \%$ e de 35,6 a 9,1\%, respectivamente frente a $C$. acutatum. 
Oliveira et al. (2019) encontraram percentagem de inibição frente a cepa de Colletotrichum theobromicola igual a $68,80 \%$ na maior concentração usual, e na menor concentração de $17 \%$ do OE de Zingiber officinale. Já no estudo desenvolvido por Martinazzo et al. (2019) onde avaliaram o OE de Cymbopogon citratus em diferentes concentrações frente à Aspergillus flavus. Os pesquisadores encontraram melhor atividade fungistática nas concentrações de 1,0; 0,8 e $0,6 \mu \mathrm{L}^{-1}$ com atividade antifúngica entre 100 a $34 \%$, e nas menores concentrações de 0,4 e 0,2 $\mu \mathrm{L}^{-1}$ com inibição entre 100 a $11 \%$.

Fiori et al. (2000) comprovaram a eficiência dos OEs de Achillea millefolium, Cymbopogon citratus, Eucalyptus citriodora e Ageratum conyzoide frente ao desenvolvimento micelial de D. bryoniae. 0 citral extraído do $\mathrm{OE}$ de Cymbopogon citratus apresenta grande potencial contra o desenvolvimento micelial das espécies Candida albicans, A. citri, Aspergillus fumigatus, A. oryzae, F. oxysporum, F solani, H. compactum, Sclerotium rolfsii, T. mentagrophytes e Rhizoctonia solani (Gonçalves, 2012; Freddo et al., 2016). 0 citral pode ser obtido de inúmeras espécies vegetais, em Lippia alba este composto apresentou atividade fúngica em Aspergillus fumigatus e Candida krusei (Mesa-Arango et al., 2009).

Os OEs também apresentam em especial, atividade bactericida frente às inúmeras bactérias (gram positivas e negativas), bem como no controle de alguns vírus causadores de doenças em vegetais, animais e humanos (Allahverdiyev et al., 2004; Tariq et al., 2019).

Resultados relevantes obtidos pelo uso de diversos OEs, com importantes taxas de rendimento e atividades biológicas no controle de fitopatógenos de origem fúngica, como observado neste estudo para os frutos verde e maduro de Fortunella margarita vem demonstrando a necessidade de se usarem os compostos extraídos in natura dos vegetais como forma alternativa no controle e erradicação de inúmeras fitopatologias avaliadas em meio in vitro e em casas-de-vegetação. Ambos os OEs de F. margarita apresentaram como possíveis dois novos agentes antifúngicos para o controle e erradicação de $S$. sclerotiorum, C. gloeosporioides e C. acutatum que todos os anos causam sérios problemas de perdas agrícolas em todo o mundo.

\section{AGRADECIMENTOS}

Ao Instituto Federal Goiano, Campus Rio Verde; a Universidade Federal de Jataí; a Universidade Federal de São Carlos; aos laboratórios de Química de Produtos Naturais e Química Tecnológica; as agencias de financiamento de pesquisa CAPES, FINEP, CNPq e FAPEG pela bolsa de mestrado em Agroquímica para o primeiro autor.

\section{REFERÊNCIAS BIBLIOGRÁFICAS}

Adams, R.P. 2007. Identification of essential oil components by gas chromatography/mass spectrometry. 4th Edition, Allured Publishing Corporation, 362 South Schmale Road, Carol Stream, Illinois, USA.

Allahverdiyev, A.; Duran, N.; Ozguven, M.; Koltas, S. 2004. Antiviral activity of the volatile oils of Melissa officinalis L. against Herpes simplex vírus type-2. Phytomedicine, 11(78): 657-661. DOI: https://doi.org/10.1016/j. phymed.2003.07.014

Amselem, J.; Cuomo, C.A.; van Kan J.A.; Viaud, M.; Benito, E.P.; Couloux, A.; Coutinho, P.M.; de Vries, R.P.; Dyer, P.S.; Fillinger, S.; Fournier, E. 2011. Genomic analysis of the necrotrophic fungal pathogens Sclerotinia sclerotiorum and Botrytis cinerea. PLOS Genetics, 7(8): e1002230. DOI: https://doi.org/10.1371/ journal.pgen.1002230 
Araújo, A. de.; Sousa, N.R. 1998. Doenças numa coleção diversificada de recursos genéticos de espécies autóctones da Amazônia. Manaus: EMBRAPA - CPAA. Boletim de Pesquisa, 6. 14pp.

Boland, G.J.; Hall, R. 1994. Index of plant hosts of Sclerotinia sclerotiorum. Canadian Journal of Plant Pathology, 16(2): 93-108.

Botelho, P.de.S.; Morais, M.M.de.; Nevez, I.de.A.; Neves, R.C.S.; Ribeiro, N.de.C.; Born, F.de.S.; Da Camara, C.A.G. 2009. Composição química e ação repelente do óleo essencial da laranja lima (Citrus aurantium L.) sobre o ácaro rajado Tetranychus urticae Kock. In: IX Jornada de Ensino, Pesquisa e Extensão, Recife - PE.

Bragança, C.A.D.; Damma, U.; Baroncelli, R.; Massola Júnior, N.S.; Crous, P.W. 2019. Species of the Colletotrichum acutatum complex associated with anthracnose diseases of fruit in Brazil. Fungal Biology, 120(4): 547-561. DOI: https://doi.org/10.1016/j.funbio.2016.01.011 Chagas, A.C.de.S.; Passos, W.M.; Prates, H.T.; Leite, R.C.; Furlong, J.; Fortes, I.C.P. 2002. Efeito acaricida de óleos essenciais e concentrados emulsionáveis de Eucalyptus spp. em Boophilus microplus. Brazilian Journal of Veterinary Research Animal Science, 5: $247-$ 253. DOI: https://doi.org/10.1590/S141395962002000500006

Cruz, M.J.D.S.; Clemente, E.; Cruz, M.E.da.S.; Mora, F.; Cossaro, L.; Pelisson, N. 2010. Effects of bioactivenatural compounds on the postharvest conservation of mango fruits cv. Tommy Atkins. Ciência e Agrotecnologia, 34(20): 428433. DOI: http://dx.doi.org/10.1590/S141370542010000200022

Cunico, M.M.; Cirio, G.M.; Miguel, O.G.; Miguel M.D.; Montrucchio, D.P.; Auer, C.G.; Grigoletti Júnior, A. 2002. Contribuição ao estudo da atividade antifúngica de Maytenus ilicifolia Mart. ex Reiss., Celastraceae. Revista Brasileira de
Farmacognosia, 12(2): 69-73. DOI: https://doi. org/10.1590/S0102-695X2002000200003

Damm, U.; Sato, T.; Alizadeh, A.; Groenewald, J.Z.; Crous, P.W. 2019. The Colletotrichum dracaenophilum, C. Magnum and C. orchidearum species complexes. Studies in Mycology, 92: 1-46. DOI: https://doi.org/10.1016/j. simyco.2018.04.001

Dann, E.K.; Ploetz, R.C.; Coates, L.M.; Pegg, K.G. 2002. Foliar, fruit and soilborne diseases. In: Whiley, A. W.; Schaffer, B.; Wolstenholme, B.N. (Eds.). The avocado: botany, production and uses. CAB International, Wallingford, UK, p. 299-338.

Da Silva, T.K.; Borges, B.G.; De Freitas, A.S.; Soares, M.G.de.O.; Freitas, E.J.; Alcantra, E.; Figueiredo, J.R.M. 2018. Atividade antifúngica in vitro de própolis sobre Colletotrichum spp. do abacate. Revista da Universidade Vale do Rio Verde, 16(3): 1-6. DOI: http://dx.doi.org/10.5892/ ruvrd.v16i3.5607

De Morais, S.M.; Júnior, F.E.A.C.; Da Silva, A.R.A.; Neto, J.S.M.; Rondina, D.; Cardoso, J.H.L. 2006. Atividade antioxidante de óleos essenciais de espécies de Croton do nordeste do Brasil. Revista Química Nova, 29(5): 907910. DOI: https://doi.org/10.1590/S010040422006000500004

De Souza, R.L.; Mesquita, F.R.; Alves, W.F. 2019. Avaliação da atividade antifúngica dos óleos essenciais de andiroba e copaíba e suas diferentes combinações no controle do fungo Sclerotium rolfsii. Scientia Naturalis, 1(1): 1725.

Dias-Arieira, CR.; Ferreira, L.da.R.; Arieira, J.de.O.; Miguel, E.G.; Donega, M.A.; Ribeiro, R.C.F. 2010. Atividade do óleo de Eucalyptus citriodora e Azadirachta indica no controle de Colletotrichum acutatum em morangueiro. Summa Phytopathological, 36(3): 228232. DOI: https://doi.org/10.1590/S010054052010000300007 
Diniz, A.B.; De Oliveira, D.R. 2015. Chemical composition of kinkan orange and citrus fruits. Demetria, 10(4): 835-844. DOI: http://dx.doi. org/10.12957/demetra.2015.16726

Do Carmo, L.T.; Sotão, H.M.P.; De Brito, F.M.; Moura, M.F.; De Oliveira, J.R. 2016. Riqueza de fungos causadores de ferrugens em plantas hospedeiras da região metropolitana de Belém, PA, Brasil. Hoehnea, 43(4): 557-573. DOI: https://doi.org/10.1590/2236-8906$07 / 2016$

Donadio, L.C.; Mourão-Filho, F.A.A.; Moreira, C.S. 2005. Centros de origem, distribuição geográfica das plantas cítricas e histórico da citricultura no Brasil. In: Mattos Júnior, D.; De Negri, J.D.; Pio, R.M., Pompeu Júnior, J. (Eds) Citros, Campinas: Instituto Agronômico e Fundag, p.1-18.

Ferreira, A.S.; Warren, H.L. 1982. Resistance of Sorghum to Colletotrichum graminicola. Plant Disease, 66: 773-775.

Fiori, A.C.G.; Schwan-Estrada, K.R.F.; Stangarlin, J.R.; Vida, J.B.; Cruz, M.E.S.; Pascholati, S.F. 2000. Antifungal activity of leaf extracts and essential oils of some medicinal plants against Didymella bryoniae. Journal of Phytopathology, 148: 483487. DOI: https://doi.org/10.1046/j.14390434.2000.00524.x

Fitsiou, E.; Mitropoulou, G.; Spyridopoulou, K.; Tiptiri-Kourpeti, A.; Vamvakias, M.; Bardouki, H.; Panayiotidis, M.I.; Galanis, A.; Kourkoutas, Y.; Chlichlia, K.; Pappa, A. 2016. Phytochemical profile and evaluation of the biological activities of essential oils derived from the greek aromatic plant species Ocimum basilicum, Mentha spicata, Pimpinella anisum and Fortunella margarita. Molecules, 21(8): 1069. DOI: https://doi.org/10.3390/ molecules21081069

Freddo, Á.R.; Lewandowski, A.; Busso, C.; Cechim, F.E.; Zorzzi, I.C.; Rey, M.dos.S.; Dalacosta, N.L.; Mazaro, S.M. 2016. Óleo essencial de
Aloysia citriodora no controle de Sclerotinia sclerotiorum em pepino e atividade antifúngica in vitro. Cultura Agronômica, 25(4): 373386. DOI: https://doi.org/10.32929/24468355.2016v25n4p373-386

Garcia, R.Á.; Juliatti, F.C.; Barbosa, K.A.G.; Cassemiro, T.A. 2012. Atividade antifúngica de óleo e extratos vegetais sobre Sclerotinia sclerotiorum. Bioscience Journal, 28 (1): 48-57. Gonçalves, A.H. 2012. Atividade fungitóxica dos óleos essenciais de Lippia sidoides Cham. e de Cymbopogon citratus (D.C.) Stapf. No controle de fitopatógenos do feijoeiro. Dissertação de mestrado. Universidade Federal do Tocantins. 90pp.

Koyasako, A.; Bernhard, R.A. 1983. Volatile constituents of the essential oil of Kumquat. Journal of Food Sciences, 48(6): 1807-1812.

Lopes, J.M.S.; Déo, T.F.G.; Andrade, B.J.M.; Giroto, M.; Felipe, A.L.S.; Júnior, C.E.I.; Bueno, C.E.M.S.; Silva, T.F.; Lima, F.C.C. 2011. Importância econômica do citros no Brasil. Revista Científica Eletrônica de Agronomia, 20: 1-2.

Lopes, L.T.A.; De Paula, J.R.; Tresvenzol, L.M.F.; Bara, M.T.F.; Sá, S.de.; Ferri, P.H.; Fiuza, T.de.S. 2013. Composição química e atividade antimicrobiana do óleo essencial e anatomia foliar e caulinar de Citrus limettioides Tanaka (Rutaceae). Revista de Ciências Farmacêuticas Básica e Aplicada, 34(4): 503-511.

Ladaniya, M. 2008. Citrusfruit: biology, techonology and evaluation. Academic Press, 558pp.

Martinazzo, A.P.; Oliveira, F.da.S.de.; Teodoro, C.E.de.S. 2019. Antifungal activity of Cymbopogon citratus essential oil against Aspergillus flavus. Ciência e Natureza, 41(e20): 7-8. DOI: 10.5902/2179460X36055

Martins, F.T.; Dos Santos, M.H.; Polo, M.; Barbosa, L.Cde.A. 2006. Variação química do óleo essencial de Hyptis suaveolens (L.) Poit., sob 
condições de cultivo. Química Nova, 29(6): 1203-1209.

Mazzini, R.B.; Pio, R.M. 2010. Caracterização morfológica de seis variedades cítricas com potencial ornamental. Revista Brasileira de Fruticultura, 32(2): 463-470. DOI: https://doi. org/10.1590/S0100-29452010005000043

Mendes, L.B.; Bedin, C.; Lopes, R.L.B.; Bosquê, G.G. 2007. Ocorrência da Sigatoka negra em banana (Musacea spp.). Revista Científica de Agronomia, 7(12).

Mesa-Arango, A.C.; Montiel-Ramos, J.; Zapata, B.; Durán, C.; Betancur-Galvis, L.; Stashenko, E. 2009. Citral and carvone chemotypes from the essential oils of Colombian Lippia alba (Mill.) N. E. Brown: composition, citotoxity and antifungal activity. Memórias do Instituto Oswaldo Cruz, 104(6): 878-884. DOI: https://doi. org/10.1590/S0074-02762009000600010

Millezi, A.F.; Baptista, N.M.; Caixeta, D.S.; Rossoni, D.F.; Cardoso, M.G.; Piccoli, R.H. 2014. Caracterização química e atividade antibacteriana de óleos essenciais de plantas condimentares e medicinais contra Staphylococcus aureus e Escherichia coli. Revista Brasileira de Plantas Medicinais, 16(1): 18-24. DOI: https://doi.org/10.1590/S151605722014000100003

Miranda, C.A.S.F.; Cardoso, M.das.G.; Batista, L.R.; Rodrigues, L.M.A.; Figueiredo, A.C.da.S. 2016. Óleos essenciais de folhas de diversas espécies: propriedades antioxidantes e antibacterianas no crescimento de espécies patogênicas. Revista Ciência Agronômica, 47(1): 213220. DOI: https://doi.org/10.5935/18066690.20160025

Monteiro, R.de.L.; Nogueira, S.R.; Macedo, P. 2017. Estratégias para o controle da antracnose em açaí solteiro (Euterpe precatória) no Acre. In: XXVI Seminário de Iniciação Científica da UFAC. p. 557.
Nascimento, P.F.C.; Nascimento, A.C.; Rodrigues, C.S.; Antoniolli, Â.R.; Santos, P.O.; Júnior, A.M.B.; Trindade, R.C. 2007. Atividade antimicrobiana dos óleos essenciais: uma abordagem multifatorial dos métodos. Revista Brasileira de Farmacognosia, 17(1): 108113. DOI: https://doi.org/10.1590/S0102695X2007000100020

Oliveira, S.da.S.; Hanada, R.E.; Brito, R.S.de. 2019. Composição quimica e atividade antifúngica do óleo essencial de Zingiber officinale Roscoe sobre Colletotrichum theobromicola, causador da antracnose da cebolinha (Allium fistulosum). Scientia Naturalis, 1(1): 32-40.

Pombo, J.C.P.; Ribeiro, E.R.; Pinto, R. de L.; Da Silva, B.J.M. 2018. Efeito antimicrobiano e sinergístico de óleos essenciais sobre bactérias contaminantes de alimentos. Revista Segurança Alimentar e Nutricional, 25(2): 108-117. DOI: https://doi.org/10.20396/san.v25i2.8651785 Quijano, C.E.; Pino, J.A. 2009.Volatile compounds of round Kumquat (Fortunella japônica Swingle) peel oil from Colombia. Journal of Essential Oil Research, 21(6): 483-485. DOI: https://doi.org /10.1080/10412905.2009.9700224

Rocha, J.S.; Soares Filho, W.S. 2009. Desenvolvimento de variedades-copa híbridas de citros: plantas ornamentais. In: Anais da Jornada Científica Embrapa Mandioca e Fruticultura Tropical, 3. Cruz das Almas. Cruz das Almas: Embrapa Mandioca e Fruticultura Tropical.

Santos, T.G.; Rebelo, R.A.; Dalmarco, E.M.; Guedes, A.; Gasper, A.L.de.; Cruz, A.B.; Schmit, A.P.; Cruz, R.C.B.; Steindel, M.; Nunes, R.K. 2012. Composição química e avaliação da atividade antimicrobiana do óleo essencial das folhas de Piper malacophyllum (C. Presl.) C. DC. Química Nova, 35(3): 477-481. DOI: https://doi. org/10.1590/S0100-40422012000300007

Sicari, V.; Poiana, M. 2017. Comparison of the volatile component of the essential oils of 
Kumquat (Fortunella margarita Swingle) extracted by supercritical carbon dioxide, hydrodistillation and conventional solvent extraction. Journal of Essential Oil Bearing Plants, 20(1): 87-94. DOI: https://doi. org/10.1080/0972060X.2017.1282841

Silva, EAJ.; Da Silva, V.P.; Alves, C.C.F.; Alves, J.M.; Souchie, E.L.; Barbosa, L.C.A. 2018. Chemical composition of the essential oil of Psidium guajava leaves and its toxicity against Sclerotinia sclerotiorum. Semina: Ciências Agrárias, 39(2): 865-874. DOI: http://dx.doi. org/10.5433/1679-2018v39n2p865

Siviero, A.; Moreira G.T.S.; Macedo, P.E.F.; Nogueira, S.R. 2019. Doenças do cacau e do cupuaçu no Acre. In: 51o Congresso Brasileiro de Fitopatologia, Recife. p. 451

Solórzano-Santos, F.; Miranda-Novales, M.G. 2012. Essential oils from aromatic herbs as antimicrobial agents. Current Opinion in Biotechnology, 23(2): 136-141. DOI: https:// doi.org/10.1016/j.copbio.2011.08.005

Sutour, S.; Luro, F.; Bradesi, P.; Casanova, J.; Tomi, F. 2016. Chemical composition of the fruit oils of five Fortunella species grown in the same pedoclimatic conditions in Corsica (France). Natural Product Communications, 11(2): 259262.

Tariq, S.; Wani, S.; Rasool, W.; Shafi, K.; Bhat, M.A.; Prabhakar, A.; Shalla, A.H.; Rather, M.A. 2019. A comprehensive review of the antibacterial, antifungal and antiviral potential of essential oils and their chemical constituents against drug-resistant microbial pathogens. Microbial Pathogenesis, 134: 103580. DOI: https://doi. org/10.1016/j.micpath.2019.103580

Xavier, MN.; Alves, J.M.; Carneiro, N.S.; Souchie, E.L.; Silva, E.A.J.; Martins, C.H.G.; Ambrósio, M.A.L.V.; Egea, M.B.; Alves, C.C.F.; Miranda, M.L.D. 2016. Composição química do óleo essencial de Cardiopetalum calophyllum Schltdl. (Annonaceae) e suas atividades antioxidante, antibacteriana e antifúngica. Revista Virtual de Química, 8(5).

Wang, Y.W.; Zeng, W.C.; Xu, P.Y.; Lan, Y.J.; Zhu, R.X.; Zhong, K.; Huang, Y.N., Gao, H. 2012. Chemical composition and antimicrobial activity of the essential oil of Kumquat (Fortunella crassifolia Swingle) peel. International Journal of Molecular Science, 13(3): 3382-3393.

Recibido: 8 de noviembre de 2019 Aceptado para publicación: 25 de febrero de 2020

Esta obra está bajo una Licencia Creative Commons Atribución-NoComercial-SinDerivar 4.0 Internacional. 